\title{
Abdominal nonparenchymatous cystic lesions and their mimics in children
}

\author{
Kaan Esen · Anıl Özgür · Yasemin Karaman · \\ Hakan Taşkınlar • Meltem Nass Duce • \\ F. Demir Apaydın · Taylan Kara
}

Received: 12 June 2014/ Accepted: 15 August 2014/Published online: 9 September 2014

(C) Japan Radiological Society 2014

\begin{abstract}
Abdominal cystic lesions in children may originate from parenchymatous organs or from nonparencyhmatous structures. Although these lesions have welldescribed imaging features, proper diagnosis usually depends on the accurate determination of the origin of the lesion. Because large lesions may resemble each other it is difficult to identify the site of origin, which results in a diagnostic dilemma. In this pictorial essay we describe abdominal nonparenchymatous cystic lesions and their mimics arising from parenchymatous organs in children.
\end{abstract}

Keywords Cystic lesion · Nonparenchymatous ·

Parenchymatous $\cdot$ Children $\cdot$ Abdominal

\section{Introduction}

Abdominal cystic lesions of childhood have a wide range of differential diagnosis. These lesions may be classified as parenchymatous or nonparenchymatous, on the basis of their site of origin. Parenchymatous cystic lesions arise from solid organs whereas nonparenchymatous lesions originate from the bowel, mesentery, omentum, and

K. Esen $(\bowtie) \cdot$ A. Özgür · M. N. Duce · F. Demir Apaydın ·

T. Kara

Department of Radiology, Mersin University Faculty of

Medicine, 34. Cadde, Mersin, Turkey

e-mail: kaanessen@yahoo.com

Y. Karaman

Department of Radiology, Antalya Education and Research

Hospital, Antalya, Turkey

H. Taşkınlar

Department of Pediatric Surgery, Mersin University Faculty of Medicine, Mersin, Turkey retroperitoneum. The term "mesenteric cyst" is used to define cysts within the mesentery, including lymphatic malformations, enteric duplication cysts, nonpancreatic pseudocysts, and mesothelial cysts [1].

Although clinical issues such as patient age, symptoms, physical signs, and laboratory findings are helpful in diagnosis, imaging is essential. Ultrasonography (US) is the imaging tool used for initial evaluation of abdominal cystic lesions in children because of its widespread availability and lack of ionizing radiation. Cross-sectional modalities, for example magnetic resonance imaging (MRI) or computed tomography (CT), may provide additional information enabling detailed definition and characterization of the lesions.

The first stage of definitive diagnosis is to identify the origin of the lesion. When the lesion is within the borders of a solid organ, differential considerations are usually limited to the pathologies arising from the parenchyma of this organ. Diagnosis of mesenteric or omental cyst may easily be established if the lesion arises strictly from a solid organ. However, especially for a large mass, determining the site of origin may be difficult [2]. In such cases, a huge parenchymatous cyst may easily be confused with a mesenteric or omental cyst.

In this pictorial essay, abdominal cystic masses of childhood, and their mimics, which originate from nonparenchymatous tissues are described.

\section{Nonparenchymatous cystic lesions}

Meconium pseudocyst

Meconium pseudocyst is a meconium peritonitis-related intraperitoneal cystic cavity which results from perforation 

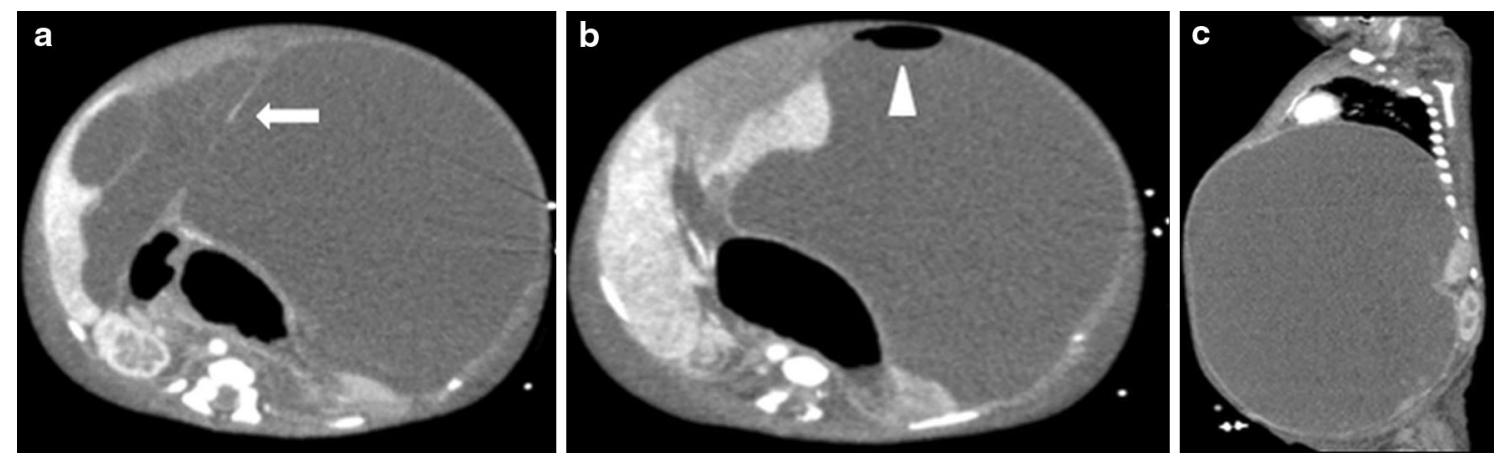

Fig. 1 Meconium pseudocyst in a newborn. a, b, c Axial and sagittal CT images show a giant cystic mass filling the abdomen and displacing the bowel loops laterally. Note the presence of septation (arrow) and air (arrowhead), suggestive findings that aid diagnosis of meconium pseudocyst

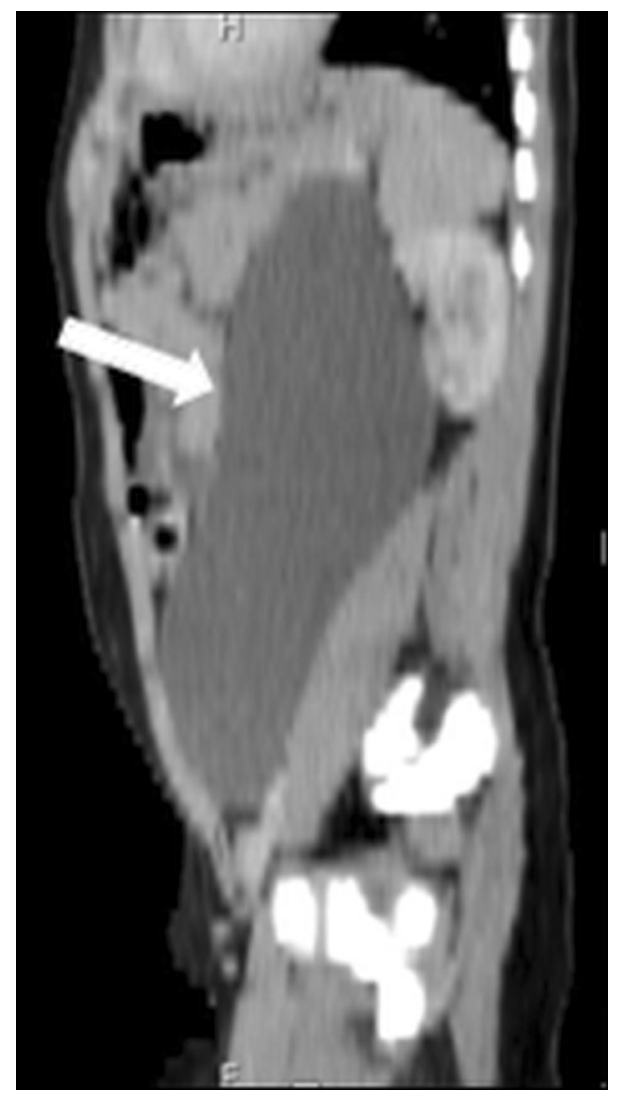

Fig. 2 Mesothelial cyst in a 8-year-old girl. Sagittal CT image shows an abdominal thin-walled cystic mass without any septation or solid component (arrow)

of the intestine in the neonatal or prenatal period. The perforation may be because of obstruction secondary to atresia, stenosis, meconium ileus, volvulus, Meckel's diverticulum, peritoneal bands, and meconium plug syndrome in cystic fibrosis. The clinical symptoms are abdominal distention, acidosis, and vomiting [3]. US reveals a large hypoechoic lesion filling the abdomen. Intraluminal air, wall calcification, and communication with a dilated bowel loop are important imaging features
(Fig. 1). On CT, findings similar to those of US are detected; however, CT may be helpful in revealing wall calcification.

\section{Mesothelial cyst}

Mesothelial cysts are rare mesenteric cysts lined with mesothelial cells. Diagnosis may be difficult because of nonspecific clinical presentation and imaging findings. Mesothelial cysts are thin-walled and contains serous material [1]. Imaging modalities reveals a cystic mass which may be giant and displace the adjacent organs (Fig. 2).

Cystic retroperitoneal and sacrococcygeal teratoma

Teratomas are neoplasms arising from three embryonic germ cell layers. Although teratomas may develop in different sites of the body, the sacrococcygeal region and the ovary are the most common locations. Retroperitoneal location is rare in children. Nevertheless, sacrococcygeal teratoma is one of the most common congenital tumors. Clinical findings of teratomas depend on the size and the location of the tumor. Most teratomas are predominantly cystic but imaging findings are variable, with solid, multicystic, or heterogeneous appearance $[4,5]$. US shows an anechoic or hypoechoic cystic mass with internal hyperechoic areas indicative of such ectodermal components as teeth, hair, fat, and calcification [6]. Fat, fat-fluid level, and calcifications are important clues that can be seen in most of these lesions (Fig. 3); the presence of fat is the major characteristic of teratomas. CT or MRI are ideal for the demonstration of ectodermal components (Fig. 4).

\section{Lymphatic malformation}

Lymphatic malformation is a congenital malformation of the lymphatic system that usually occurs in the head and neck region. Abdominal and thoracic lymphatic malformations 

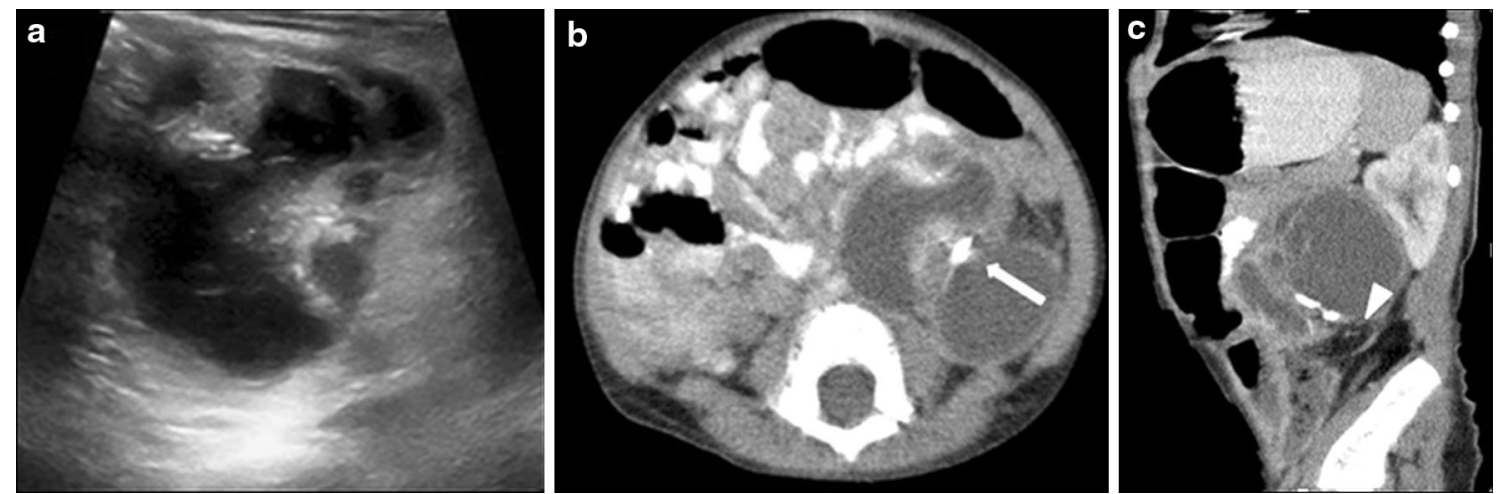

Fig. 3 Retroperitoneal teratoma in a 4-year-old girl. a US shows a heterogeneous cystic mass containing solid component. b, $\mathbf{c}$ On CT, a retroperitoneally located, heterogeneous, thick-walled cystic mass is seen. The mass contains septations, a solid component, calcifications (arrow), and fat (arrowhead)
Fig. 4 Cystic sacroccocygeal teratoma in a 9-year-old girl. a Sagittal T2-weighted MR image shows a giant hyperintense lesion in the presacral location occupying the pelvis. b On axial contrast enhanced fat saturated T1weighted MR image the lesion is hypointense without any contrast enhancement. The rectum is anteriorly displaced by the mass (arrow)
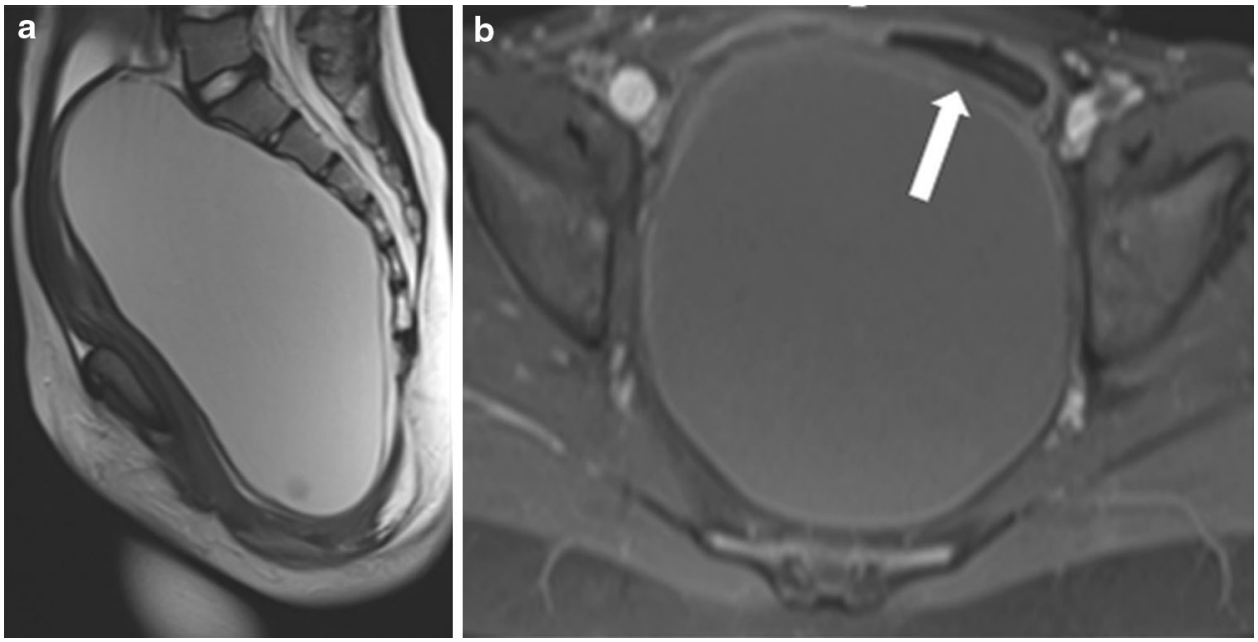

are rare. Abdominal lymphatic malformation is frequently seen in a mesenteric location, and is the most commonly encountered mesenteric cyst [1, 7]. Lymphatic malformation may be confused with other cystic lesions, because of its diverse clinical and imaging findings. Patients may present with abdominal distention, lymphoedema in the lower extremities, or symptoms related to intestinal obstruction. The typical imaging finding is a unilocular or multilocular interseptated and/or interconnecting thin-walled cystic lesion (Fig. 5). Cysts are frequently homogeneous and anechoic on US but also may contain echogenic debris. Diagnosis may be difficult if the lesions are complicated with hemorrhage or infection. In retroperitoneal lymphatic malformations, intercompartmental extension may be seen as a characteristic elongated shape [7].

Gastrointestinal duplication cyst

Gastrointestinal (GI) duplication cysts are congenital lesions that can occur anywhere within the GI tract; the

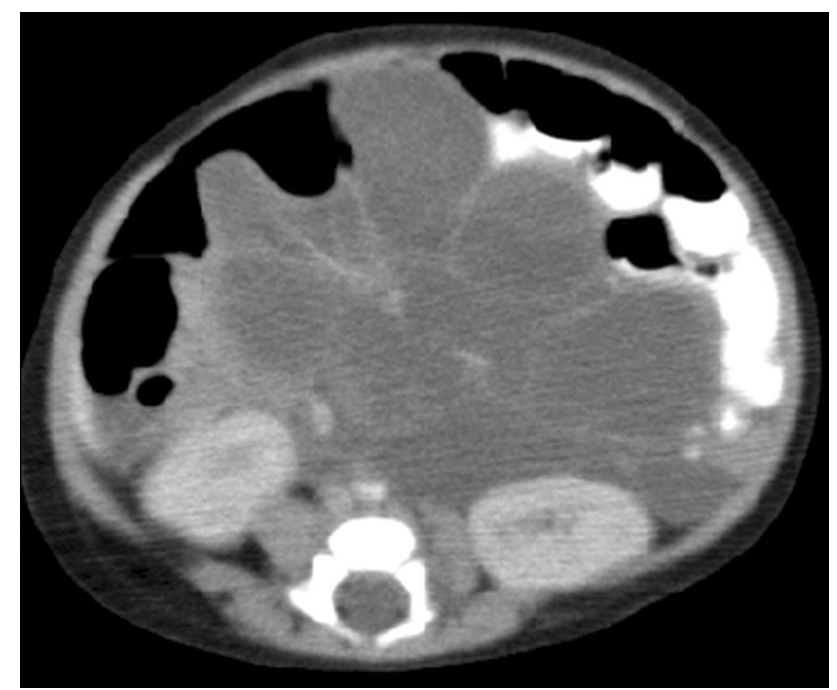

Fig. 5 Lymphatic malformation in a 1-year-old girl. Transverse CT image shows a multilocular, interseptated cystic mass in the abdomen 


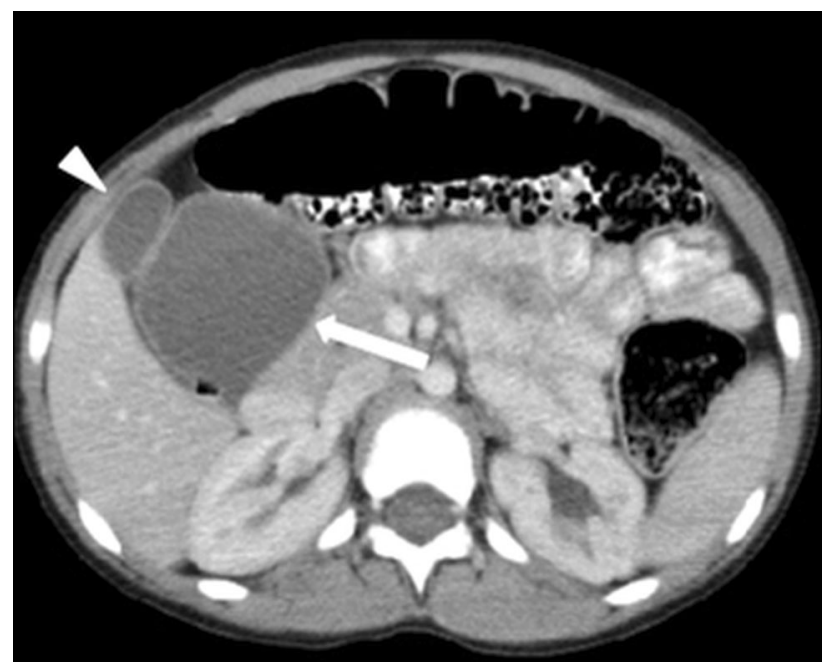

Fig. 6 Gastrointestinal duplication cyst in a very rare location, the pyloroduodenal region, in a 6-year-old girl. Axial CT shows a right upper quadrant cystic mass anterior to the duodenum (arrow). The gall bladder is anterior to the cyst (arrowhead)

ileum is the most common site. They contain GI epithelium, arise from the mesenteric side and may communicate with the GI tract or may be completely isolated $[2,6]$. Obstruction, pain, and GI bleeding are the major clinical symptoms depending on the location and size of the lesion. On US, hypoechoic or anechoic lesions adherent to the GI tract are detected (Fig. 6). Inner echogenic mucosa surrounded by a hypoechoic rim is an important clue for diagnosis. It may contain internal debris as a result of hemorrhage or mucosal secretions. CT shows a hypodense lesion on the mesenteric side of the GI tract. Scintigraphy can be useful for diagnosis of duplication cysts with gastric mucosa [2].

\section{Cerebrospinal fluid pseudocyst}

Cerebrospinal fluid (CSF) pseudocysts are rare complications of ventriculoperitoneal shunting. Incidence ranges from 1 to $4.5 \%$ [8]. Patients present with abdominal distention, pain, and with symptoms related to an increase of intracranial pressure. These are cystic masses around the shunt tip, without any epithelial lining on the wall. US reveals a well-defined anechoic cystic mass. Internal echoes or septa may be a result of pseudocyst infection [2, 8]. $\mathrm{CT}$ is more useful for showing the relationship between the cyst and the catheter tip (Fig. 7).

\section{Choledochal cyst}

Choledochal cysts are cystic dilatations of the biliary ductal system. They are believed to be related to abnormal insertion of the common bile duct into the pancreatic duct,

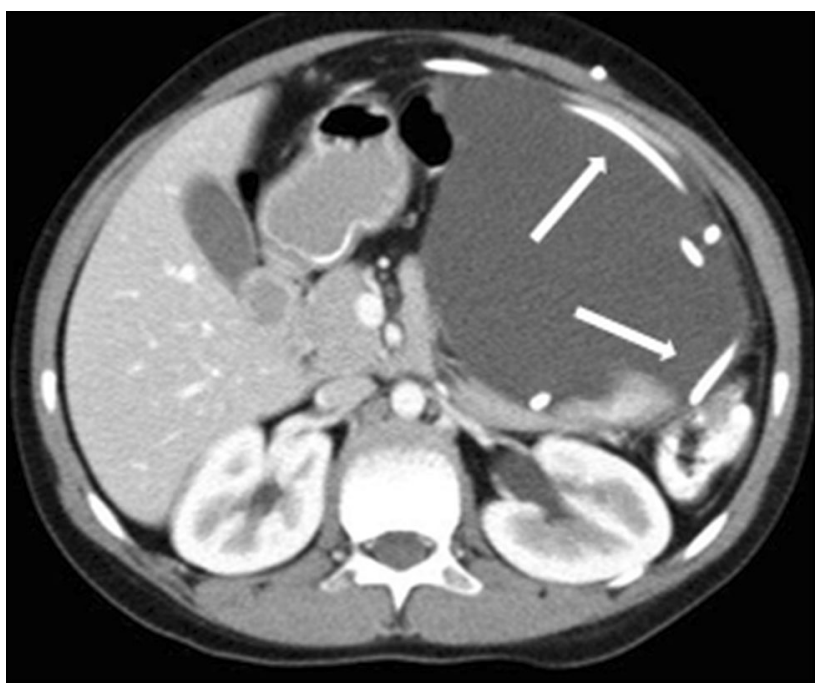

Fig. 7 CSF pseudocyst in a 9-year-old boy. CT shows a cystic mass in the left part of the abdomen; the ventriculoperitoneal shunt catheter is within the cyst (arrows)

which causes chemical cholangitis and dilatation of the biliary tree. Classic symptoms, for example abdominal mass, jaundice, and pain, are seen in $25 \%$ of patients only [2]. There are five different types of choledochal cysts in the Todani classification system and type 1 is the most frequent. The Todani classification is:

- Type 1: Segmental or diffuse fusiform dilatation of the extrahepatic bile duct

- Type 2: True diverticulum of an extrahepatic bile duct

- Type 3: Choledochocele

- Type IVa: Multiple extrahepatic and intrahepatic bile duct cysts

- Type IVb: Multiple extrahepatic bile duct cysts

- Type 5: Single or multiple cystic dilatations of the intrahepatic bile ducts (Caroli disease)

A cystic mass in the hepatic hilus, separate from the gall bladder, is the most common imaging finding (Fig. 8). When a choledochal cyst is suspected sonographically, MRCP and scintigraphy should be performed to reveal communication of the cyst with the ductal system. Nevertheless, other causes of biliary duct dilatations, for example choledochal stone and tumor should be ruled out. Cholelithiasis or choledocholitiasis, pancreatitis, and malignancy are the common complications of choledochal cysts [9].

\section{Abscess}

Abscess is the localized accumulation of pus and infected fluid. Intraabdominal abscesses are rare in children and can be located anywhere in the abdomen, for example parenchymatous organs, mesentery, and retroperitoneum. The 
Fig. 8 Choledochal cyst in a 5-month-old girl. a, b Axial and coronal contrast-enhanced CT show a giant hypoattenuating cystic mass at porta hepatis and dilated intrahepatic bile ducts (arrow)
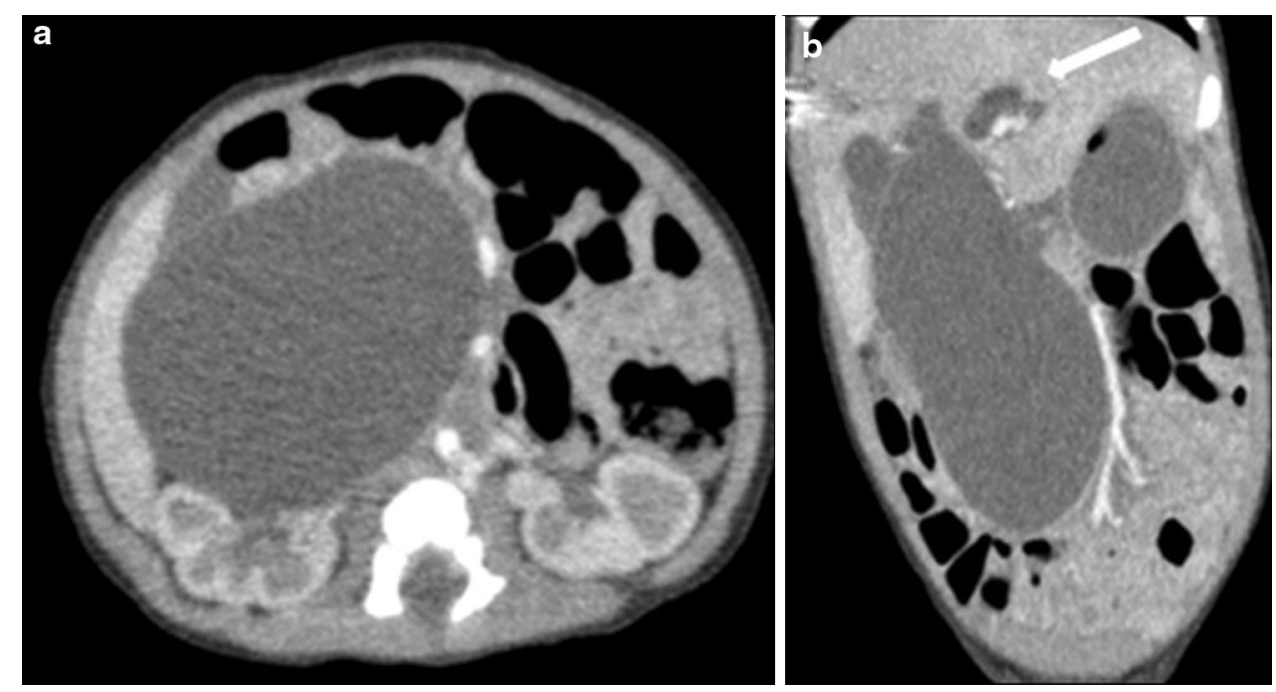

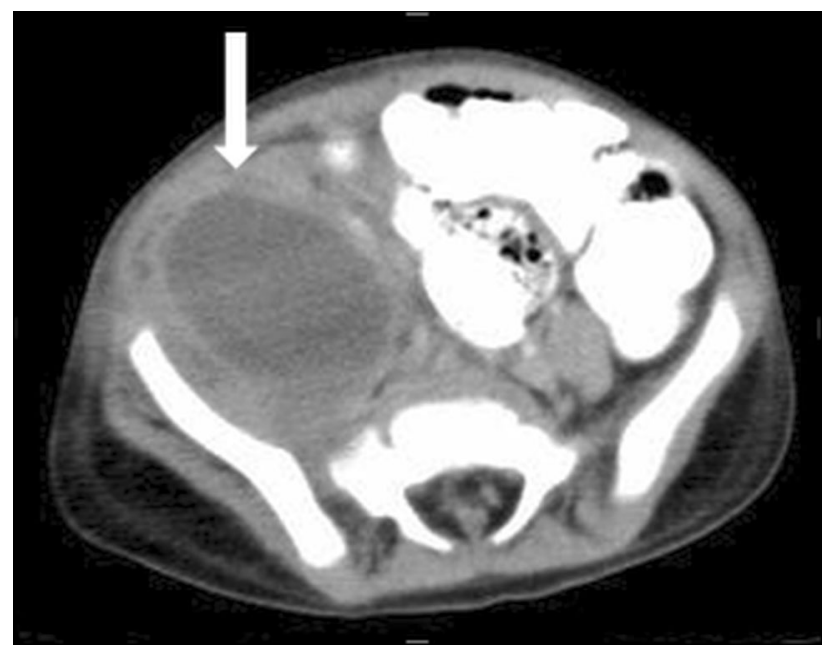

Fig. 9 Iliopsoas abscess in a 2-year-old girl. Axial CT shows a hypodense cystic lesion in the right side of the pelvis (arrow). Note that the adjacent fat planes are obliterated

most common cause of abdominal or pelvic abscess in children is appendicitis; postsurgical, posttraumatic, and Crohn disease-related abscesses may also be seen (Fig. 9), [10]. Iliopsoas muscle is an uncommon location for abscess formation but it may easily be confused with diseases such as appendicitis, because of its variable clinical findings (Fig. 10).

Patients generally present with pain, fever, nausea, and abdominal distention. Imaging findings are variable, depending on the location and age of the abscess. On US, abscesses may have a wide range of appearance from hypoechoic to heterogeneous-hyperechoic. However, CT is the preferred imaging modality especially for retroperitoneal lesions when US is inefficient because of bowel gas. On CT, abscesses are usually seen as hypodense cystic lesions with rim-like contrast enhancement; some lesions

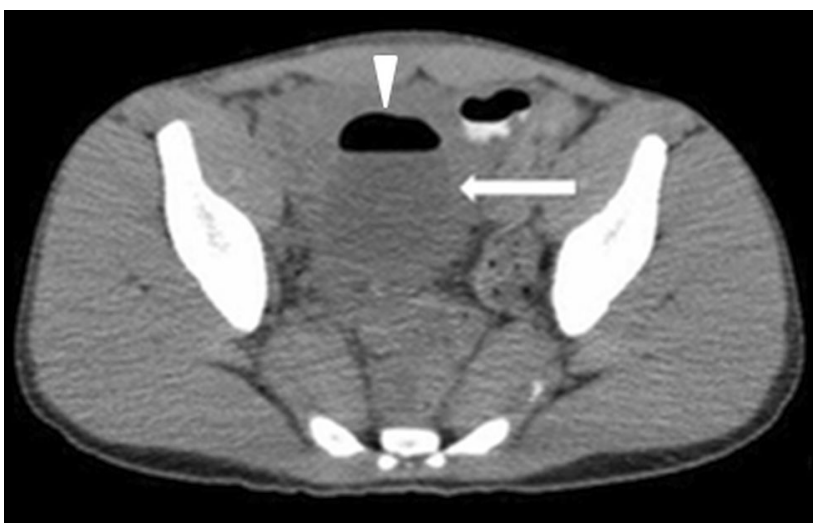

Fig. 10 Abscess after appendectomy in a 16-year-old boy. Axial CT shows a well-defined cystic mass in the pelvis (arrow) including air (arrowhead)

may manifest as soft tissue masses, however. Wall thickening in the adjacent intestine and obliteration or stranding of fat planes around the abscess may also be detected.

\section{Mimics}

Mesenchymal hamartoma

Mesenchymal hamartoma is an uncommon, developmental malformation of liver composed of hepatocytes, bile ducts, mesenchyme, portal vein branches, and cysts [2]. It is a benign tumor commonly occurring in the first 2 years of life and is usually large. Mesenchymal hamartomas are most commonly located in the right lobe of the liver; however, approximately $20 \%$ are pedunculated. In this instance, they are located outside the liver parenchyma and can mimic a nonparenchymatous abdominal cystic lesion 


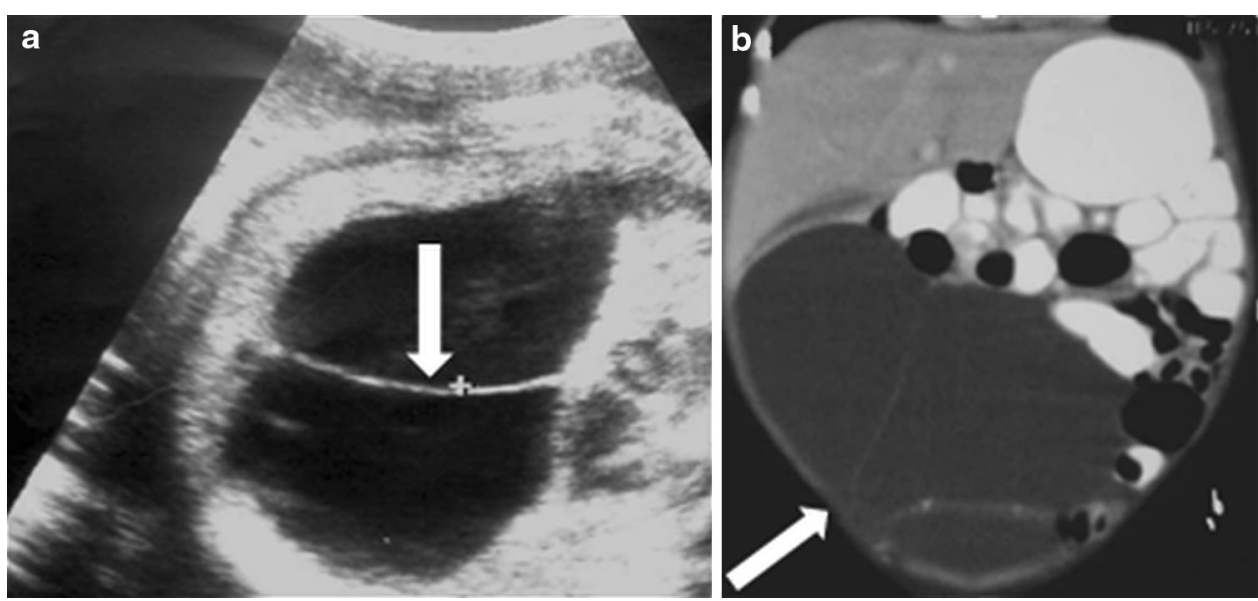

Fig. 11 Mesencymal hamartoma in a male newborn. a US shows a huge cystic mass with internal septations (arrow). b Coronal contrastenhanced CT shows a $9 \times 6 \mathrm{~cm}$ cystic mass filling the right side of abdomen and pelvis, in close vicinity to the liver (arrow). Note the lack of demonstrable continuity between the mass and the liver. Radiological prediagnosis was omental or mesenteric cyst. However, a pedunculated liver mass was found during surgery. The histopathological diagnosis is mesenchymal hamartoma of liver
(Fig. 11), [2]. US should be the primary screening modality. Mesenchymal hamartoma is predominantly cystic; multiseptated cystic appearance is typical. It may also be solid with internal calcifications. In the differential diagnosis of solid lesions, tumors such as hepatoblastoma must be included $[11,12]$.

\section{Cystic neuroblastoma}

Neuroblastoma is the second most common abdominal mass among children. It originates from the neural crest in the adrenal medulla or in any place where the sympathetic ganglion is located. Although US is useful in primary diagnosis, CT or MRI is superior in demonstrating the extent of the lesion and staging of the disease. On imaging, a heterogeneous soft tissue mass with small cystic areas caused by hemorrhage or necrosis is seen. Calcifications are present in $80-90 \%$ of patients and CT is better for revealing these [13, 14]. Cystic forms are uncommon compared with solid forms.

\section{Ovarian cyst}

Ovarian cysts are common in childhood and may be classified as functional, physiological, or neoplastic. Physiological and functional cysts include follicle and corpus luteal cysts; the most common ovarian neoplasms in children are teratoma and cystadenoma [2, 6]. Abdominal distention and pelvic pain are major symptoms of ovarian cysts. US is the initial method of choice for evaluation of cystic lesions and for differentiating these from solid cysts.

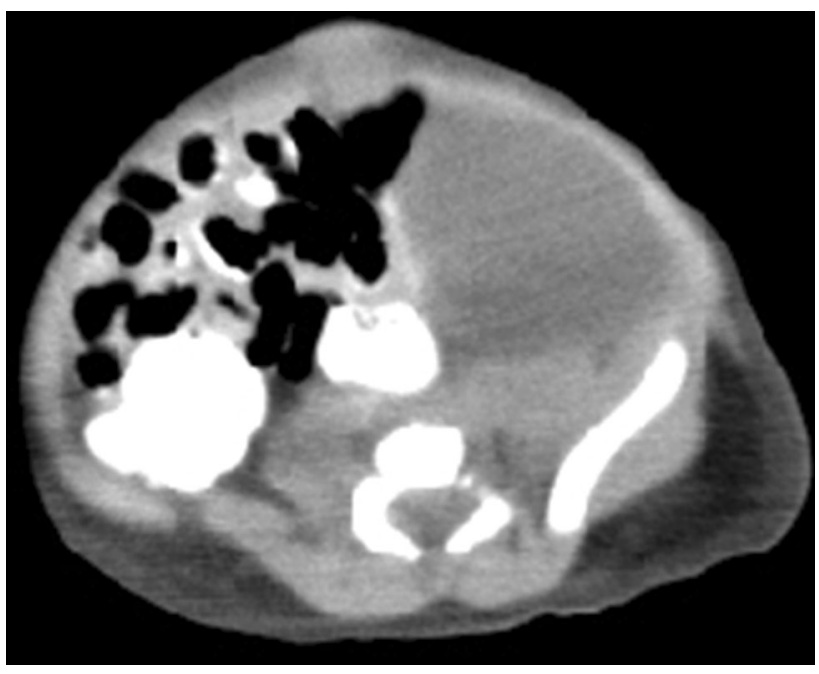

Fig. 12 Ovarian cyst in a 1-year-old girl. Axial CT shows a hypodense cystic mass in the left side of the pelvis. Note the lack of septations or solid component within the mass

On US, an anechoic thin-walled cystic mass, which may extend cranially to the abdomen, is typical for simple cystic lesions (Fig. 12). Hemorrhage, rupture, and torsion are the most common complications. Complicated ovarian cysts may include internal echoes, septa, and heterogenous clots. Contrast enhancement of the wall and septa of ovarian cysts may be observed on CT. Nevertheless, solid components and nodular septa are important indicators of malignancy; if this is suspected, MRI should be performed, because of its multiplanar capability and high soft-tissue resolution. 


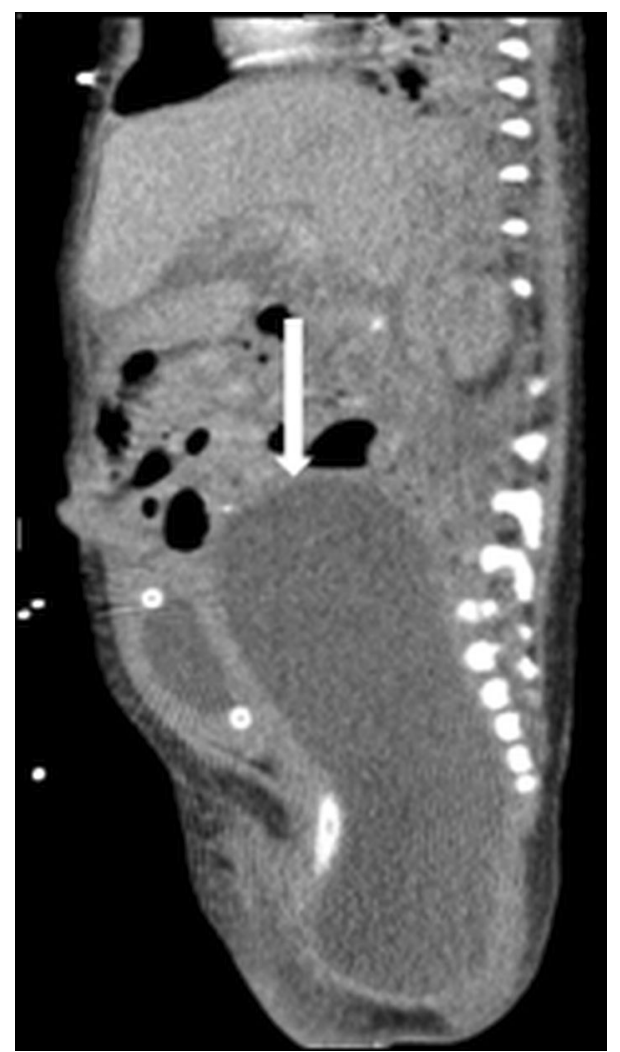

Fig. 13 Hydro/hematometrocolpos in a newborn. Sagittal CT reveals a large hypodense pelvic retrovesical cystic mass extending from the vulva to the abdomen

\section{Hydro/hematometrocolpos}

Hydro/hematometrocolpos is dilatation of uterin and vaginal cavities with blood or fluid that develops because of vaginal atresia, transverse vaginal septum, and imperforate hymen. In the newborn it can be associated with urogenital sinus or cloacal malformation [15]. On US, an anechoic or hyperechoic pelvic midline mass posterior the bladder is identified, and MRI can be used to better reveal the location, extension, and cystic structure (Fig. 13).

\section{Conclusion}

Cystic abdominal masses in children may be parenchymatous or nonparencyhmatous in origin, with a wide differential diagnosis list. Diagnosis of nonparenchymatous abdominal cystic masses and their mimics is difficult because of the absence of characteristic clinical and imaging findings. Appropriate preference of imaging modalities and careful evaluation of such imaging features as internal structure, anatomic location, relationship with adjacent organs, and extension of the lesion, with clinical history and the patient's age, will enable accurate diagnosis.

Conflict of interest The authors declare that they have no conflicts of interest.

\section{References}

1. Stoupis C, Ros PR, Abbitt PL, Burton SS, Gauger J. Bubbles in the belly: imaging of cystic mesenteric or omental masses. RadioGraphics. 1994;14:729-37.

2. Wootton-Gorges SL, Thomas KB, Harned RK, Wu SR, SteinWexler R, Strain JD. Giant cystic abdominal masses in children. Pediatr Radiol. 2005;35:1277-88.

3. Valladares E, Rodríguez D, Vela A, Cabré S, Lailla JM. Meconium pseudocyst secondary to ileum volvulus perforation without peritoneal calcification: a case report. J Med C Rep. 2010;4:292.

4. Xu Y, Wang J, Peng Y, Zeng J. CT characteristics of primary retroperitoneal neoplasms in children. Eur $\mathrm{J}$ Radiol. 2010;75:321-8.

5. Ueno T, Tanaka YO, Nagata M, Tsunoda H, Anno I, Ishikawa S, et al. Spectrum of germ cell tumors: from head to toe. RadioGraphics. 2004;24:387-404.

6. Onur MR, Bakal U, Kocakoc E, Tartar T, Kazez A. Cystic abdominal masses in children: a pictorial essay. Clin Imaging. 2013;37:18-27.

7. Levy AD, Cantisani V, Miettinen M. abdominal lymphangiomas: imaging features with pathologic correlation. AJR. 2004;182:1485-91.

8. Pernas JC, Catala J. Pseudocyst around ventriculoperitoneal shunt. Radiology. 2004;232:239-43.

9. Kim OH, Chung HJ, Choi BG. Imaging of the choledochal cyst. Radiographics. 1995;15:69-88.

10. Brown C, Kang L, Kim ST. Percutaneous drainage of abdominal and pelvic abscesses in children. Semin Intervent Radiol. 2012;29:286-94.

11. Stanley P, Hall TR, Woolley MM, Diament MJ, Gilsanz V, Miller JH. Mesenchymal hamartomas of the liver in childhood: sonographic and ct findings. AJR. 1986;147:1035-9.

12. Kim SH, Kim WS, Cheon JE, Yoon HK, Kang GH, Kim IO, et al. Radiological spectrum of hepatic mesenchymal hamartoma in children. Korean J Radiol. 2007;8:498-505.

13. Elsayes KM, Mukundan G, Narra VR, Lewis JS, Shirkhoda A, Farooki A, et al. Adrenal masses: mr imaging features with pathologic correlation. RadioGraphics. 2004;24:73-86.

14. Papaioannou G, McHugh K. Neuroblastoma in childhood: review and radiological findings. Cancer Imaging. 2005;5:116-27.

15. Garel L, Dubois J, Grignon A, Filiatrault D, Vliet GV. US of the pediatric female pelvis: a clinical perspective. RadioGraphics. 2001;21:1393-407. 